\title{
Hedgehog signalling as a target in cancer stem cells
}

\author{
Vanessa Medina $^{1}$, Moisés B. Calvo ${ }^{2}$, Silvia Díaz-Prado ${ }^{2,3}$, Jesús Espada $^{4}$ \\ ${ }^{1}$ Oncology Research Unit, University Hospital A Coruña, A Coruña, Spain \\ ${ }^{2}$ Biomedical Research Institute of A Coruña (INIBIC), A Coruña, Spain \\ ${ }^{3}$ Medicine Department, University of A Coruña, A Coruña, Spain \\ ${ }^{4}$ Biomedical Research Institute "Alberto Sols", CSIC-UAM, Madrid, Spain
}

\begin{abstract}
Hedgehog $(\mathrm{Hh})$ is one of the most important signalling pathways. Together with the Wnt, TGF- $3 / \mathrm{BMP}$ and Notch pathways, it is involved in both embryonic development and adult tissue homeostasis. This is because Hh plays a central role in the proliferative control and differentiation of both embryonic stem cells and adult stem cells. In this way, an alteration in the Hh pathway, either by misexpression of components of that pathway or by changes in the expression of other cellular components that interfere with the Hh signalling system, may trigger the development of several types of cancer. This occurs because normal stem cells or their intermediaries toward differentiated mature cells are not part of the normal proliferative/differentiation balance and begin to expand without control, triggering the generation of the so-called cancer stem cells. In this review, we will focus on the molecular aspects and the role of Hh signalling in normal tissues and in tumour development.
\end{abstract}

Keywords

Hh (Hedgehog); Smoothened (Smo); Patched (Ptc); Gli ; Cancer; Cancer stem cells

\section{Introduction}

An important molecular mechanism that provides cell differentiation is signal transduction. The signal transduction pathways ensure the reception of the concentration gradients of morphogens and their transformation into the differentiated state of cells within tissues and organs. Hence it may be assumed that at the molecular level the key molecular rearrangements may be related to changes in genes that participate in signal transduction pathways.

The Hedgehog (Hh) cascade, its sensors, switches and routers have been amply described and studied [1]. It is involved in diverse morphogenetic processes. A set of proteins accomplishing versatile functions can be distin-guished in the $\mathrm{Hh}$ signal cascade. These include the $\mathrm{Hh}$ morphogen, the Ptc and Smo cell receptors, the Cos2, Pka, Slmb, Su(Fu) and Fu proteins that make up the high-molecular-weight complex transducing the Hh signal within the cell nucleus, and the $\mathrm{Ci}$ transcription factor. It is known how these proteins interact in the functioning Hhcascade and the interactions can be described as a gene network. A computer-based description has been deposited in the Gene Net database [2]. 


\section{Evolutionary history}

Hh signal transduction has startling parallels with Wnt signalling, despite the different structures of the ligands and the largely distinct components that are dedicated to the separate pathways.

As both pathways are found throughout the animal kingdom, a common ancestral pathway (or at least components that contributed to both pathways) must have been present in the earliest metazoans.

The structure of the Hh molecule itself is very similar to that of zinc hydrolases and other enzymes, including bacterial ones. This similarly has fuelled speculation that the Hh signalling system is derived from an ancient metabolic pathway [3]. There are further interesting parallels between bacterial processes and signalling in animals. The inner bacterial membrane contains several translocators with an overall 12-transmembrane topology. Some of these translocators serve as efflux pumps to clear toxic drugs from the cell. These pumps contain multiple subunits including a 12-transmembrane molecule of the resistance-nodulation-cell division (Rnd) family, a proton-driven molecular transporter. The overall organisation of the Rnd proteins is similar to that of Ptc/Disp and there is conservation of some essential amino acids between these molecules [4]. Ptc may control the translocation of small molecules over membranes in mammalian cells [4]. Another class of translocators in the bacterial membrane belongs to the $\mathrm{ABC}$ transporter family, ATP-driven translocators. These molecules also have 12 transmembrane domains, but their topology and sequence appears to be different from the Rnd/Ptc family.

However, the function of these Abc transporters is reminiscent of Disp: they translocate lipidmodified proteins over the inner membrane into the periplasmic space. Moreover, many bacterial lipoproteins carry palmitate as their lipid, covalently linked to a cysteine at the $\mathrm{N}$ terminus of the protein. Among the functions of bacterial lipoproteins is cell-to-cell communication, as exemplified by the Tgl protein in Myxococcus. Hence, a signalling system based on lipid-modified proteins and specific membrane translocators is ancient, and may have been the founder of the Wnt and Hh signalling systems.

\section{Structural features}

The Hh proteins are destined for secretion. Because molecules that are secreted from cells are commonly glycosylated but not, as far as is known, acylated, the discovery that Wnt and $\mathrm{Hh}$ proteins carry covalently attached palmitates [5] came as a surprise (Fig. 1). In the case of $\mathrm{Hh}$, palmitoylation is one of two lipid modifications, the other one being cholesterol. The Hh protein is made as a precursor molecule, consisting of a $\mathrm{C}$-terminal protease domain and an $\mathrm{N}$-terminal signalling unit, and undergoes a number of unusual modifications during its synthesis. The $\mathrm{N}$ terminus of Hh becomes modified by the fatty acid palmitate, on a conserved cysteine residue that is exposed at the very $\mathrm{N}$-terminal end of the protein after its signal sequence has been removed. The palmitoyl group is attached through an amide to the $\mathrm{NH} 2$ group of the cysteine, but it is thought that the initial link between palmitate and the cysteine is a thioesther to the sulphydryl group, after which the palmitate is transferred to the $\mathrm{NH} 2$ terminus [6].

The processing of $\mathrm{Hh}$ is unusual in a number of ways: $\mathrm{Hh}$ is made initially as a precursor molecule that consists of a $\mathrm{C}$-terminal protease domain and an $\mathrm{N}$-terminal signalling unit. The $\mathrm{C}$ terminal protease of $\mathrm{Hh}$ cleaves the precursor in an autocatalytic manner to release the active signalling domain of $\mathrm{Hh}$ called HhNp. During this cleavage, the $\mathrm{C}$ terminus of $\mathrm{HhNp}$ becomes covalently modified by a cholesterol molecule [7]. Various forms of Hh have been engineered that lack the autocatalytic cleavage site and are not therefore cholesterol-modified. These variants are called $\mathrm{HhN}$; the mammalian forms of $\mathrm{HhN}$ appear not only to lack cholesterol but are also much less efficiently palmitoylated [6]. Assuming that the relationship between cholesterol modification and palmitoylation is general and also pertains to Drosophila, this should be considered in 
interpreting the relative contributions of the cholesterol and palmitate modifications to Hh activity in vivo and in different genetic backgrounds.

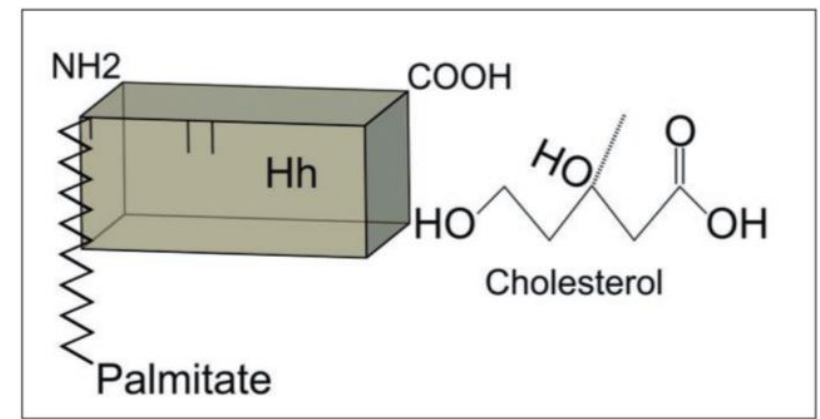

Fig. 1. The Hh protein showing the approximate positions of cysteine residues. Hh is palmitoylated through an amide on the $\mathrm{N}$-terminal cysteine. The two other cysteines in Hh are not disulphide linked and have free sulphydryl groups

\section{Function}

\section{Transport and release of Hedgehog}

Lipid attachment is a common modification of cytoplasmic proteins and is important for the membrane targeting of intracellular signalling molecules. However, as far as is known, it is rare in proteins that operate outside cells. It has also been reported that Hh protein can act on cells away from their source, as concentration-dependent, long-range morphogenetic signals. The release of Hh from cells requires a dedicated transport molecule: a protein called Dispatched (Disp). Initially found in Drosophila but functionally conserved in mammals, Disp is a multiple-pass, transmembrane protein. In the absence of Disp, Hh is not secreted from cells and is unable to signal to neighbouring cells.

Interestingly, non-cholesterol modified $\mathrm{HhN}$ is not de-pendent on Disp; it is secreted and is fully active, suggesting that the primary function of Disp is to transport cholesterol-modified Hh [8]. However, as mentioned above, it should be kept in mind that $\mathrm{HhN}$ also lacks palmitate [6]; it therefore remains possible that Disp is specifically needed for the release of palmitoylated $\mathrm{Hh}$ from cells. It is not clear how the palmitate influences Hh transport from one cell to another. Variants of Hh that lack the palmitoylation site are secreted from cells, perhaps more efficiently than wild-type $\mathrm{Hh}$ is [6]. The same is true for Hh protein in the absence of rasp [9]; however, the non-palmitoylated Hh protein is not functional.

The palmitate on $\mathrm{Hh}$ is attached to a cysteine, but through an amide at the $\mathrm{N}$ terminus, which leaves the sulphydryl group free. Thus, the lack of palmitate on Hh (in the rasp mutant) does not change the overall number of free sulphydryl groups. In fact, none of the three cysteines in Hh are involved in disulphide formation.

Evidence has been presented for a freely diffusible form of Sonic Hh (Shh) that is cholesterol modified and multimeric [10].

Much work in this area remains to be done, but as answering these questions is central to our understanding of developmental mechanisms in general, there will no doubt be significant interest in further experimental tests. 
Cells employ multiple receptors to receive instructions from $\mathrm{Hh}$ signals, in complex and little understood configurations (Fig. 2). Remarkably, the Frizzled (Fz) receptors for Wnts are related to the Smoothened (Smo) protein that is necessary for Hh signalling. Both receptors have seven transmembrane domains and a long $\mathrm{N}$-terminal extension called a cysteine-rich domain (CRD). As a group, these molecules are more closely related to each other than they are to the other families of serpentine receptors, of which there are many.

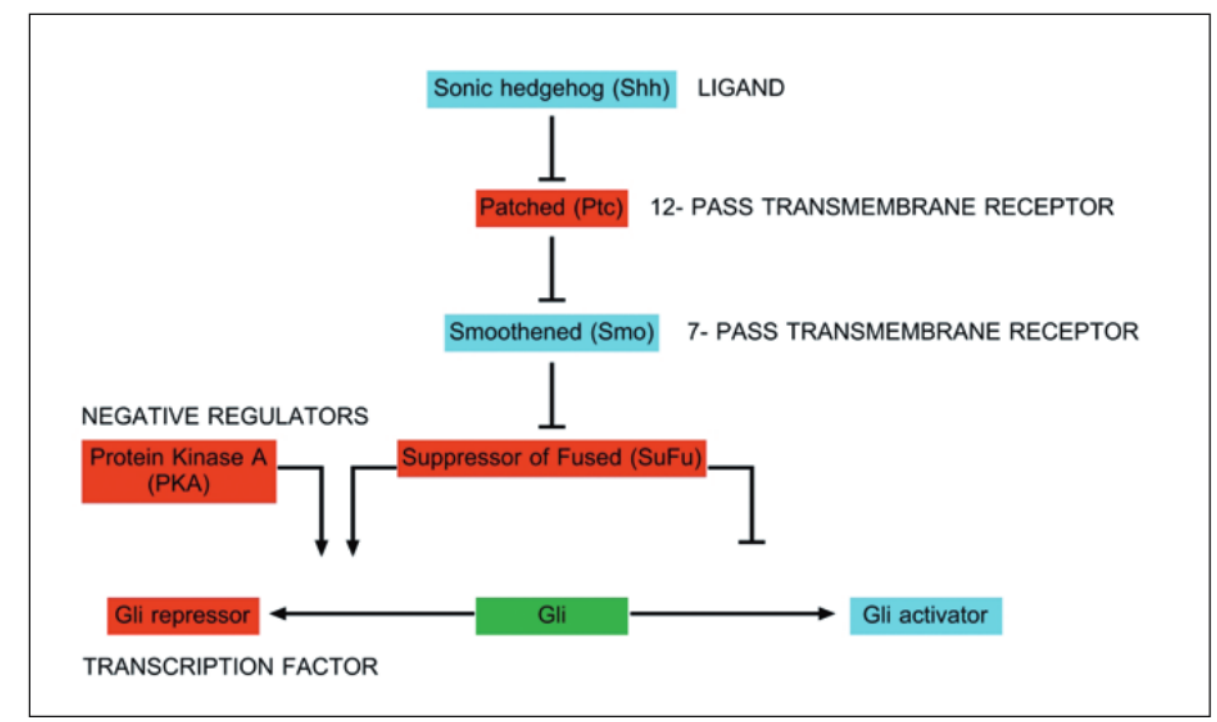

Fig. 2. Basic structure of mammalian Hh signalling pathway. Positive regulators are shown in blue boxes and negative regulators in red boxes. Abbreviations for the proteins used throughout the text are given in parentheses. Only the core, most extensively studied proteins in the pathway are shown

Although $\mathrm{Fz}$ and Smo are related to each other, it should be stressed that the actual mechanisms of activation of $\mathrm{Fz}$ and Smo are fundamentally different. Smo is not thought to interact with an extracellular ligand, whereas Wnt proteins bind directly to the CRD of Fz [11]. The way in which Hh engages with its receptor has been subject to debate, but a consensus has emerged in which Hh ligands, Sonic, Indian and Desert Hh in vertebrates and Hh in Drosophila, bind to Patched (Ptc) (Fig. 2), a 12-pass transmembrane protein. As a consequence of this interaction, Smo is activated, or to be more precise, is released from an inhibitory activity that is exerted by Ptc when Ptc is not engaged by a ligand. In other words, the negative interaction between Ptc and Smo is relieved by binding of Hh to Ptc, which turns Ptc activity off. Inhibition of Smo by Ptc may not be by direct binding, but rather by a catalytic activity of Ptc [4].

As Smo can be both activated and inhibited by small molecules, it has been suggested that Ptc might act as the transporter of an endogenous small compound that interacts with Smo [12]. Smo can be activated to a constitutive, i.e., Ptc-independent, state by specific point mutations found in tumours [12] and it is thought that these mutant forms of Smo are refractory to inhibition by small molecules.

In mammals a protein called megalin may control the endocytic uptake of the Hh protein by acting as a direct binding partner [13]. Moreover, mouse embryos lacking megalin are phenotypically similar to $\mathrm{Hh}$ mutants. Megalin is an LDL receptor-related protein (it is also called Lrp2), and is therefore related to Lrp5 and Lrp6, the Wnt interacting members of the family. 


\section{The Hedgehog signalling pathways}

The Hh signalling pathway influences the transcription of many target genes, which vary between tissues, cell types and phyla. It participates in the development of numerous tissues and organs. One dramatic effect of reduced Hh signalling in human embryos is cyclopia (the formation of only one eye), which is a form of holoprosencephaly. In adults, Hh signalling directs the formation or the persistence of certain stem- and precursor-cell populations. Increased $\mathrm{Hh}$ signalling in some organs can lead to distinct types of cancer (skin, cerebellum, muscle, digestive tract, pancreas or prostate). Aspects of Hh biology that have been recently reviewed include its numerous roles in development, disease and cancer; its unique mode of signal transduction; and connections with evolutionarily apparently older proteins that provide clues about the evolutionary origins of the Hh signalling pathway.

Hh signals are received, transduced and interpreted using cellular machinery that has been largely conserved during the divergent evolution of insects and mammals. Newly identified components of $\mathrm{Hh}$ signal transduction and the relationships between these components will be the focus of this review.

\section{Hedgehog signalling in vertebrates}

Hh signalling in vertebrates shares many features with that in Drosophila melanogaster, although clear distinctions have emerged. First, mammalian gene families take the place of single genes in D. melanogaster. There are three Hh genes in mammals, sonic, Indian and desert hedgehog (Shh, Ihh and Dhh); two ptc genes (Ptc1 and Ptc2); and three ci homologues (Gli1, Gli2 and Gli3).

The three Hh genes are expressed in different tissues and at different stages of development, and might also have different biological activities. The expression and function of Ptc1 is similar to that of D. melanogaster Ptc (that is, where Hh signals are received), whereas Ptc2 expression is more restricted and few phenotypes are associated with its loss [14]. The post-translational regulation of $\mathrm{Ci}$ and the GLI proteins is similar. Each resides in a cytoplasmic pool. In the absence of $\mathrm{Hh}$, each is retained in the cytoplasm by Cos2 (Kif7 in vertebrates) and Sufu to limit transcriptional activation $[15,16]$. Ci, Gli3, and probably also Gli2, require Pka and an SCF E3 ubiquitin ligase for processing to a transcriptional repressor. However, each Gli protein also has unique roles: GLI3 functions mainly as a transcription-al repressor, Gli2 is mainly a transcriptional activator and Glil functions only as a transcriptional activator.

The transcription of Gli1 is induced by Hh signals, which creates a positive regulatory loop that heightens Hh responses. The most important differences between Drosophila and vertebrate Hh pathways centre on Smo, its regulators and its effectors. The sequence of the cytoplasmic tail of Smo is highly divergent between vertebrates and Drosophila.

Differences in its phosphorylation and regulation are discussed in more detail below. The entire Kif7 protein from zebrafish has some sequence similarity to Cos2, and Kif7 can bind Gli1. Like Cos2, Kif7 is required for repression of Shh responses [17], although it might differ from Cos2 in the degree to which it is required for full activation of Hh responses. Another kinesin and two ciliary proteins (Kif3a and the intraflagellar transport proteins Ift88 and Ift172) also mediate Cos2-like functions in vertebrates, participating in both full repression and full activation of $\mathrm{Hh}$ responses.

Some vertebrate Hh pathway genes have no known orthologues in D. melanogaster; some have orthologues, the role of which in Hh signalling has not been explored; and some have known orthologues with other functions.

Missing in metastasis (Mim), which is also known as Beg4, is an actin-binding protein that potentiates Gli-dependent transcriptional activation. 
Because Mim is transcriptionally induced by Shh, it functions as a positive regulator that reinforces $\mathrm{Hh}$ target gene activation, similar to Gli1. Positive vertebrate regulators of the $\mathrm{Hh}$ signalling pathway that have no known orthologues in flies include megalin, which belongs to the low-density lipoprotein (LDL)-receptor-related family and binds Shh41, and iguana, a zinc-finger protein that promotes the nuclear localisation of Gli1 [18]. Negative regulatory factors distinguish vertebrate Hh signalling as well: Fkbp8 is a transcription factor that antagonises Shh action in the nervous system, whereas Sil is a cytosolic protein that seems to functions downstream of Ptc. Rab23 is a regulator of vesicular trafficking and a negative regulator of the Hh response [19].

Shifted (Shf) is a secreted protein and is the Drosophila orthologue of human Wnt inhibitory factor (Wif). Shf facilitates Hh signalling by binding $\mathrm{Hh}$ and heparan sulphate proteoglycans, whereas Wif binds Wnt proteins and facilitates Wnt signalling.

At least some of the apparent differences between phyla are the result of the functional convergence of non-homologous genes and proteins. The mammalian membrane glycoprotein Hhinteracting protein (Hip) and Drosophila Pxb have no sequence similarity, but they might fulfil the same function. Each is a transcriptional target of $\mathrm{Hh}$ and each participates in a negative-feedback loop that attenuates $\mathrm{Hh}$ responses (Hip through direct binding to Shh).

\section{The Hedgehog response network}

Several rapid changes in protein conformation or modification are elicited by Hh stimulation, including increased phosphorylation of $\mathrm{Smo}, \mathrm{Cos} 2, \mathrm{Fu}$ and $\mathrm{Su}(\mathrm{Fu})$, and de-creased phosphorylation of Ci (Fig. 3).

Although phosphorylation of $\mathrm{Ci}$ stimulates processing to form $\mathrm{CiR}$, the residues targeted and the functional roles of phosphorylation of other pathway components are largely undefined. These changes in phosphorylation, nevertheless, may be functionally important, because they are regulated by Ptc and Smo and are triggered by Hh stimulation.

With these phosphorylation events we can surmise that pathway activation begins within minutes after Hh stimulation. This kinetic provides constraints in considering the physical mechanisms underlying pathway activation. This cellular state, in which Smo is kept inactive, must dissipate rapidly upon Hh-mediated inactivation of Ptc. Changes in the subcellular distribution of this proposed modulator upon Hh-mediated loss of Ptc function thus should be consistent with the kinetics of pathway induction.

Similarly, critical events proposed to activate Smo, such as change in conformation, subcellular localisation, phosphorylation or dimerisation must occur on a sufficiently rapid time scale to be consistent with the kinetics of path-way activation.

One of the primary functions of Smo upon activation would be the inhibition of $\mathrm{Su}(\mathrm{Fu})$ activity through the activation of $\mathrm{Fu}$. It is tempting to speculate that $\mathrm{Su}(\mathrm{fu})$ is inactivated by phosphorylation and that this results from $\mathrm{Fu}$ activation. However, only a small fraction of $\mathrm{Su}(\mathrm{Fu})$ associ-ated with the Cos2-Fu-Ci complex is phosphorylated upon Hh stimulation, suggesting that phosphorylation may result from a transient interaction between $\mathrm{Su}(\mathrm{Fu})$ and the $\mathrm{Cos} 2-\mathrm{Fu}-\mathrm{Ci}$ complex. Mammalian $\mathrm{Su}(\mathrm{Fu})$ is capable of interact-ing with Gli proteins bound to DNA and also interacts with chromatin-modulating factors, suggesting that $\mathrm{Su}(\mathrm{Fu})$ also could affect transcriptional activity by recruiting chromatin-modulating factors to the DNA sites of Gli protein binding. 


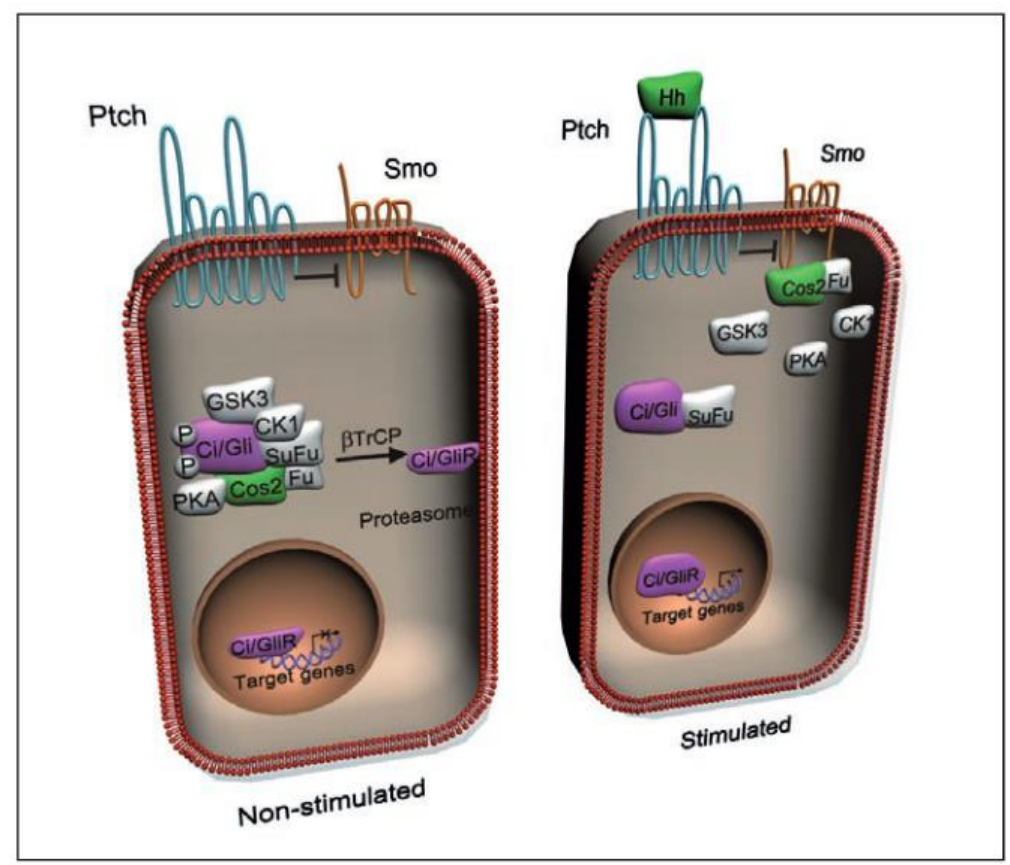

Fig. 3. Hh signalling pathway in Drosophila. In the absence of secreted Hh proteins, the Ptch receptor acts by constitutively inhibiting Smo. The Ci155 transcription factor forms a multiprotein complex with $\mathrm{Cos} 2, \mathrm{Fu}$ and $\mathrm{SuFu}$, and is sequentially phosphorylated by PKA, GSK3 and CKi protein kinases. Phosphorylated Ci155 is recognised by the Slimb/ $\beta$-TrCP component of the E3 ubiquitin ligase, ubiquitinated and targeted for degradation by the proteasome. Ci75 degraded form can translocate to the nucleus, acting as a transcriptional repressor. In the presence of $\mathrm{Hh}$ signalling, the repressor activity of the Ptch receptor is inhibited, and Smo is activated. Activated Smo blocks the phosphorylation of $\mathrm{Ci} 155$ by recruiting $\mathrm{Cos} 2$ to the cell membrane and promoting the disorganisation of the $\mathrm{Ci}$ phosphorylation complex. Ci155 can then translocate to the nucleus to activate gene transcription

\section{Hedgehog as a concentration-dependent signal}

In some tissues, including the wing of Drosophila, Hh functions as a morphogen, with different amounts of signal causing the recipient cells to adopt distinct fates. In Drosophila, the wing primordium, which is in the centre of the wing imaginal disc, is an epithelial sheet. Hh protein is secreted by posterior wing imaginal disc cells whereas Ptc is produced only by anterior cells. Anterior cells respond to Hh signals by activating the transcription of target genes that control wing growth and patterning. The overlapping (nested) pattern of target gene expression indicates that $\mathrm{Hh}$ responses are concentration dependent, a supposition that has been supported by manipulation of Hh concentrations and Ptc receptor affinities [20]. Induction of the target gene ptc requires intermediate or high concentrations of $\mathrm{Hh}$, whereas the target gene decapentaplegic (dpp) is induced by low concentrations of $\mathrm{Hh}$. This differential sensitivity is not the result of a different affinity or number of $\mathrm{Ci}$ binding sites in the respective promoters, but instead reflects differential responses to $\mathrm{CiR}$ versus $\mathrm{CiA}$.

Hh signalling stimulates the production of both antago-nists and activators, which establishes feedback loops that control the strength and duration of the response to the signal. The ptc gene is always activated by the $\mathrm{Hh}$ signal, so ptc transcription is a reliable indication of $\mathrm{Hh}$ signal reception by cells in Drosophila and vertebrates. Ptc opposes Hh action by inhibiting Smo and by binding to $\mathrm{Hh}$ and transporting to the lysosome for degradation, just as the induction of HIP production can bind and inactivate SHh. So, Hh triggers a negative-feedback loop in which the accumulation of Ptc and HIP attenuates the effect of the ongoing Hh signal. The feedback system ensures that $\mathrm{Hh}$ signalling is stable and reproducible. Any accidental reduction in the amount of 
Hh will be compensated for by reduced amounts of its antagonist proteins, whereas any increase will be balanced by higher induction of antagonists.

The lag between the induction of target genes by $\mathrm{Hh}$ and the increase in amounts of antagonist proteins might be an important timing mechanism for regulating the persistence of Hh signalling. The inducible activators Gli1 and Mim/Beg4 can counteract the effects of Ptc and Hip, at least in tissues where both an activator and an inhibitor are induced. However, in Drosophila, the Gli1 orthologue, ci, is not transcriptionally induced by the Hh signal and nothing has been reported so far about the function of the Drosophila MIM/BEG4 orthologue.

Some target genes are activated by only the highest concentrations of Hh (Fig. 4), some are activated by both intermediate and high concentrations of $\mathrm{Hh}$, and some are repressed only in the absence of $\mathrm{Hh}$. In each case, elimination of the transcriptional effector ( $\mathrm{Ci}$, or Gli2 and Gli3) generates tissue with cell types that resemble neither dorsal nor ventral tissue in the case of the neural tube and neither border nor far-anterior cells in the case of the wing.

Moreover, these phenotypes are recapitulated by loss of the cytoskeletal-association proteins (Cos2 or Kif3a/Ift) [21]. These observations illustrate that there is a fundamental similarity between Hh signalling in vertebrates and invertebrates.

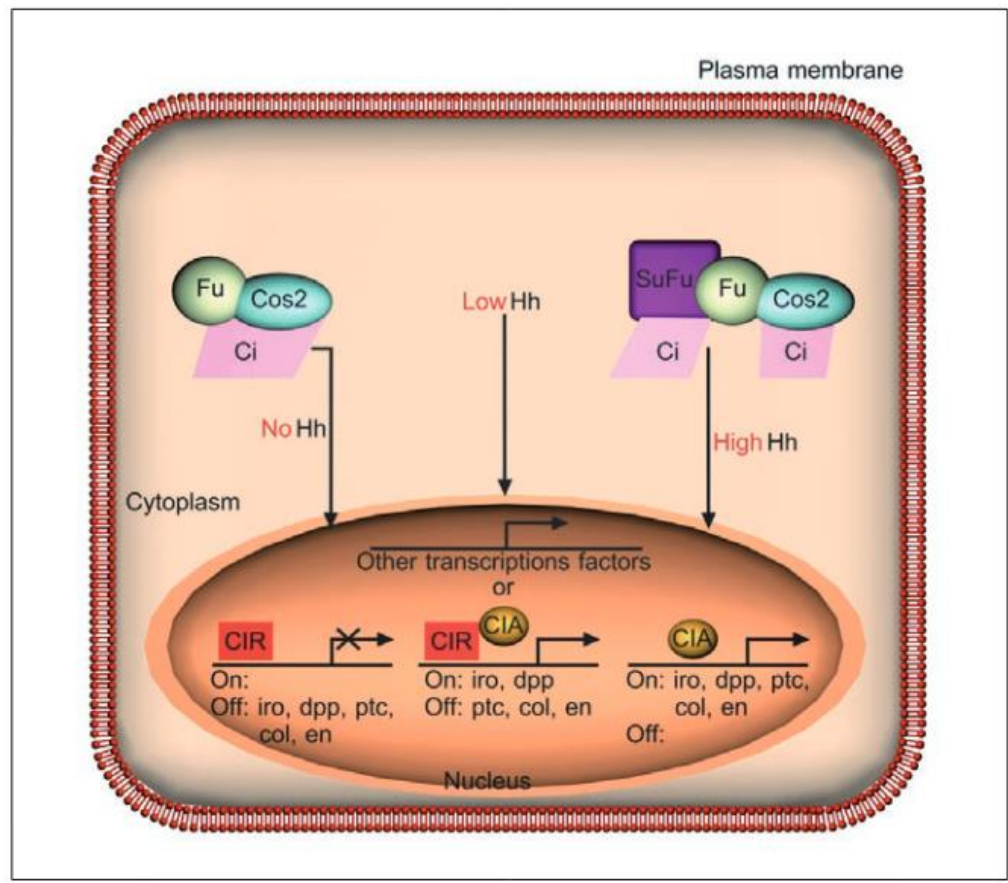

Fig. 4. Three states for the Hh pathway, for Cubitus interruptus and for Hh target genes. In the nucleus, the repressor form of Cubitus interruptus (CiR) competes with the activator form (CiA) for access to the promoter elements of Hh target genes. When $\mathrm{Hh}$ is absent, CiR predominates and transcription is blocked. When concentrations of $\mathrm{Hh}$ are high, $\mathrm{CiA}$ predominates and transcription is activated. When Hh concentrations are low, neither $\mathrm{CiR}$ nor $\mathrm{CiA}$ reaches the nucleus in significant quantities. Alternatively, similar amounts of $\mathrm{CiR}$ and $\mathrm{CiA}$ reach the nucleus. In either case, low $\mathrm{Hh}$ concentrations allow target genes to respond to transcriptional regulation by other factors. Complex I contains Fused (Fu), Costal-2 (Cos2) and Ci, whereas Complex II contains Suppressor of Fused (Sufu) and Ci 


\section{Hedgehog in development and cancer}

The Hh pathway plays a central role during embryo development to give rise to the distinct structures and organs integrated in a normal individual. This is achieved through the key action of Hh signalling on fate and proliferative capacities of distinct sets of embryonic stem cells. Moreover, in adult individuals $\mathrm{Hh}$ also plays important roles control-ling the normal self-renewal and maintenance of tissues as it is able to regulate the proliferative activity of stem cells and their differentiation toward mature cells through pro-genitor or transient-amplifying cells. For instance, in nor-mal adult tissues, expression of several components of the Hh signal transduction machinery has been detected, such as Ptc and Gli in sets of stem cells of the central nervous system and gut epithelium [22-26]. Indeed, increasing evidence indicates that the Hh pathway can be transiently reactivated by tissue damage to stimulate tissue repair, including peripheral nerve regeneration [27-29]. In this way, it has been shown that Hh signalling can regulate cell proliferation through the activation of the cell cycle regulatory proteins cyclin $\mathrm{D}$ and $\mathrm{E}$ [30]. Although less clear, the control of cell differentiation by Hh signalling might occur through the induction of protein secretion, including neurotrophic and angiogenic factors [29].

On the other hand, Hh signalling plays an important role in the morphogenesis of the intestine. Here, the ligands Sonic Hedgehog (Shh) and Indian Hedgehog (Ihh) are localised in the gut epithelial cells where paracrine communication is established with mesenchymal cells, which express the Patched-1 and Patched-2 receptors, as well as the effectors of Hh signalling, Gli1, Gli2 and Gli3.

Blocking Hh signals through the pain inhibitor Hhip (Hedgehog interacting protein) markedly compromises the crypt-villus architecture and leads to a complete absence of villi in an often pseudostratified and hyperproliferating epithelium. A milder phenotype is observed when $\mathrm{Hh}$ signalling is suppressed by the injection of an anti-Hh antibody, and is characterised by the presence of ectopic proliferation and abnormally branched villi. The general conclusion is that $\mathrm{Hh}$ signals from the epithelium to the mesenchyme are required for epithelial remodelling as well as for restraining proliferation to the intervillus regions, where the proteins Shh and Ihh become concentrated as development proceeds [31].

A similar profile in Hh signalling is observed during prostate development and its regrowth after surgical resection, where a cross-talk between proliferative epithelium and mesenchyme has been established. Despite some contradictory data, it is generally accepted that Hh signalling (both autocrine, on the same cell, and paracrine, on the surrounding mesenchymal cells) in epithelial cells located in the tips of prostate branches is associated with the production of BMP cytokines from the mesenchyme, which gives rise to an antiproliferative signal that controls branching morphogenesis. Thus, the expression of Hh ligands is mainly restricted to the developing epithelium whereas the expression of $\mathrm{Hh}$ receptors and effectors is restricted to the mesenchyme neighbour of proliferative branched epithelium (for review, see [32]).

In agreement with a key role in the regulation of cell proliferation and differentiation, several reports have indicated that aberrant activation of Hh signalling can promote cancer.

The first link between Hh signalling and cancer was the demonstration that Gorlin syndrome is the result of a PTCH1 mutation [33, 34]. Gorlin syndrome is characterised by the development of numerous basal cell carcinomas and the predisposition to other tumours, especially medulloblastoma and rhabdomyosarcoma. After that, it was shown that a large proportion of sporadic BCCs involve hyperactivation of Hh signalling after overexpression of GLI1 and PTCH1 [35-38]. Similarly, more than one third of human medulloblastomas are associated with abnormal Hh signalling linked to Ptch1 and SuFu mutations [39]. These observations have been largely corroborated in mouse models. Overexpression of Shh, Gli1, Gli2 or constitutively active Smo in the skin induces the formation of BBC-like lesions [40-42]. In the same way, mice heterozygous for a Ptch1 mutation, a mouse model of Gorlin syndrome, have a higher incidence of spontaneous medulloblastoma and are more susceptible to UV-induced BBC [43]. 
On the other hand, in mice expressing the hedgehog inhibitor Hhip, Wnt target genes that are normally restricted to the crypt compartment, such as $\mathrm{Cdx} 1, \mathrm{Cd} 44$ and EphB2, showed expression throughout the entire villus epithelium. These results indicate that the $\mathrm{Hh}$ signal negatively regulates Wnt signalling under normal conditions and that $\mathrm{Hh}$ alteration may be associated with a Wnt pathway malfunction, which may be behind the start of colorectal cancer. Indeed, in human adenomas of patients with familial adenomatous polyposis (FAP), the expression of IHH is lost in the neo-plastic tissue, where Wnt signalling is constitutively active due to a mutation of APC. Therefore, the role of $\mathrm{Hh}$ restricting Wnt signalling to the crypts seems to be lost in neoplastic conditions, which leads to aberrant Wnt activation [31].

As a whole, these results highlight the importance of Hh signalling during carcinogenesis. The association of $\mathrm{Hh}$ signalling and cancer has prompted the development of pharmacological $\mathrm{Hh}$ antagonists for the treatment of BBC, medulloblastoma and other solid tumours in preclinical models. The most widely used Hh antagonist is cyclopamine, a natural SMOH inhibitor derived from corn lilies [44]. Cell-based pharmacological screenings have identi-fied additional synthetic inhibitors of $\mathrm{SMOH}$, such as the aminoproline Cur-61414 (Curis) [44]. Both types of inhibitors can efficiently block the Hh pathway in vitro and can prevent the formation of BCC-like lesions in mouse models [44]. Cyclopamine can also significantly reduce the growth rate of mouse medulloblastoma cells both in culture and in mouse allograft models [44]. These results indicate that inhibition of Hh signalling can block tumour growth and stimulate tumour regression without side effects in normal adjacent tissues, suggesting that this therapeutic modality has a potential clinical application.

\section{Conclusions}

Our knowledge on the molecular basis of the signal transduction mechanisms involved in Hh signalling has been greatly enriched in recent years. This accumulated mass of data has permitted precise delineation of the role of this signalling pathway during embryo development from Drosophila to mammals. Also, it has become clear that the role of Hh signalling mechanisms in the adult organism is considerably more important than previously supposed. The discovery that this signalling pathway is implicated in the regulation of adult stem function has stressed the importance that homeostatic mechanisms that operate during development have for the maintenance of adult tissues. Similarly, but not surprisingly, it has been shown that the $\mathrm{Hh}$ signalling mechanism, which is essential in the regulation of stem cell proliferation and differentiation from the embryo to the adult organism, is also causally implicated in the process of carcinogenesis.

The realisation that $\mathrm{Hh}$ signalling is equally important for tumour formation has fostered efforts in the develop-ment of pharmacological inhibitors of Hh signalling with potential clinical applications. Preclinical results, in cellular in vitro or mouse models, indicate that natural or synthetic inhibitors of the Hh signalling pathway can prevent the formation and even promote the regression of tumours. However, although promising, the development of a potential clinical application of these drugs in human cancer patients is still in the very early stages and will require further efforts in basic and translational biomedicine investigation in the next years.

\footnotetext{
Acknowledgements

V.M. is supported by A Coruña University Hospital Foundation. M.B.C. is hired through the research support programme from Instituto de Salud Carlos III (Spanish Government). S.D.P. has a contract with the Isidro Parga Pondal programme from Xunta de Galicia (Spain). J.E. is a researcher hired by the Ramón y Cajal programme (Spanish Government).
} 


\section{References}

1. Lum L, Beachy PA (2004) The Hedgehog response network: sensors, switches, and routers. Science 304:1755-1759

2. Gunbin KV, Omelyanchuk LV, Ananko EA (2004) Two gene networks underlying the formation of the anterior-posterior and dorsoventral wing imaginal disc compartment boundaries in Drosophila melanogaster.In: Kolchanov NA and Hofestaedt R (eds) Proceedings of the Fourth International Conference on Bioinformatics of Genome Regulation and Structure, BGRS'2004. Institute of Cytology and Genetics Press, Vol. 2, pp 56-59

3. Taipale J, Beachy PA (2001) The Hedgehog and Wnt signalling pathways in cancer. Nature 411:349-354

4. Taipale J, Cooper MK, Maiti T, Beachy PA (2002) Patched acts catalytically to suppress the activity of Smoothened. Nature 418:892-897

5. Willert K, Brown JD, Danenberg E et al (2003) Wnt proteins are lipid-modified and can act as stem cell growth factors. Nature 423:448-452

6. Pepinsky RB, Zeng C, Wen D et al (1998) Identification of a palmitic acid-modified form of human Sonic hedgehog. J Biol Chem 273:14037-14045

7. Porter JA, Ekker SC, Park WJ et al (1996) Hedgehog patterning activity: role of a lipophilic modification mediated by the carboxy-terminal autoprocessing domain. Cell 86:21-34

8. Burke R, Nellen D, Bellotto M et al (1999) Dispatched, a novel sterol-sensing domain protein dedicated to the release of cholesterol-modified hedgehog from signalling cells. Cell 99:803-815

9. Chamoun Z, Mann RK, Nellen D et al (2001) Skinny hedgehog, an acyltransferase required for palmitoylation and activity of the hedgehog signal. Science 293:2080-2084

10. Zeng X, Goetz JA, Suber LM et al (2001) A freely diffusible form of Sonic hedgehog mediates longrange signalling. Nature 411:716-720

11. Dann CE, Hsieh JC, Rattner A et al (2001) Insights into Wnt binding and signalling from the structures of two Frizzled cysteine-rich domains. Nature 412:86-90

12. Taipale J, Chen JK, Cooper MK et al (2000) Effects of oncogenic mutations in Smoothened and Patched can be reversed by cyclopamine. Nature 406:1005-1009

13. McCarthy RA, Barth JL, Chintalapudi MR et al (2002) Megalin functions as an endocytic sonic hedgehog receptor. J Biol Chem 277:25660-25667

14. Rahnama F, Toftgard R, Zaphiropoulos PG (2004) Distinct roles of PTCH2 splice variants in Hedgehog signalling. Biochem J 378:325-334

15. Merchant M, Vajdos FF, Ultsch M et al (2004) Suppressor of fused regulates Gli activity through a dual binding mechanism. Mol Cell Biol 24:8627-8641

16. Paces-Fessy M, Boucher D, Petit E et al (2004) The negative regulator of Gli, Suppressor of fused (Sufu), interacts with SAP18, Galectin3 and other nuclear proteins. Biochem J 378:353-362

17. Tay SY, Ingham PW, Roy S (2005) A homologue of the Drosophila kinesin-like protein Costal2 regulates Hedgehog signal transduction in the vertebrate embryo. Development 132:625-634

18. Wolff C, Roy S, Lewis KE et al (2004) Iguana encodes a novel zinc-finger protein with coiled-coil domains essential for Hedgehog signal transduction in the zebrafish embryo. Genes Dev 18:1565-1576

19. Eggenschwiler JT, Espinoza E, Anderson KV (2001) Rab23 is an essential negative regulator of the mouse Sonic hedgehog signalling pathway. Nature 412:194-198

20. Mullor JL, Guerrero I (2000) A gain-of-function mutant of patched dissects different responses to the hedgehog gradient. Dev Biol 228:211-224

21. Huangfu D, Liu A, Rakeman AS et al (2003) Hedgehog signalling in the mouse requires intraflagellar transport proteins. Nature 426:83-87

22. Lai K, Kaspar BK, Gage FH, Schaffer DV (2003) Sonic hedgehog regulates adult neural progenitor proliferation in vitro and in vivo. Nat Neurosci 6:21-27

23. Machold R, Hayashi S, Rutlin M et al (2003) Sonic hedgehog is required for progenitor cell maintenance in telencephalic stem cell niches. Neuron 39:937-950

24. Palma V, Lim DA, Dahmane N et al (2005) Sonic hedgehog controls stem cell behavior in the post-natal and adult brain. Development 132:335-344

25. Palma V, Ruiz i Altaba A (2004) Hedgehog-GLI signalling regulates the behavior of cells with stem cell properties in the developing neocortex. Development 131:337-345

26. van den Brink GR, Bleuming SA, Hardwick JC et al (2004) Indian Hedgehog is an antagonist of Wnt signalling in colonic epithelial cell differentiation. Nat Genet 36:277-282

27. Mirsky R, Parmantier E, McMahon AP, Jessen KR (1999) Schwann cell-derived desert hedgehog signals nerve sheath formation. Ann N Y Acad Sci 883:196-202

28.Parmantier E, Lynn B, Lawson D et al (1999) Schwann cell-derived Desert hedgehog controls the development of peripheral nerve sheaths. Neuron 23:713-724

29. Pola R, Ling LE, Silver M et al (2001) The morphogen Sonic hedgehog is an indirect angiogenic agent upregulating two families of angiogenic growth factors. Nat Med 7:706-711

30. Duman-Scheel M, Weng L, Xin S, Du W (2002) Hedgehog regulates cell growth and proliferation by inducing Cyclin D and Cyclin E. Nature 417:299-304 
31. Madison BB, Braunstein K, Kuizon E et al (2005) Epithelial hedgehog signals pattern the intestinal cryptvillus axis. Development 132:279-289

32. Shaw A, Bushman W (2007) Hedgehog signalling in the prostate. J Urol 177:832-838

33. Hahn H, Wicking C, Zaphiropoulous PG et al (1996) Mutations of the human homolog of Drosophila patched in the nevoid basal cell carcinoma syndrome. Cell 85:841-851

34. Johnson RL, Rothman AL, Xie J et al (1996) Human homolog of patched, a candidate gene for the basal cell nevus syndrome. Science 272:1668-1671

35. Dahmane N, Lee J, Robins P et al (1997) Activation of the transcription factor Gli1 and the Sonic hedgehog signalling pathway in skin tumours. Nature 389:876-881

36. Reifenberger J, Wolter M, Weber RG et al (1998) Missense mutations in SMOH in sporadic basal cell carcinomas of the skin and primitive neuroectodermal tumours of the central nervous system. Cancer Res 58:1798-1803

37. Unden AB, Zaphiropoulos PG, Bruce K et al (1997) Human patched (PTCH) mRNA is over-expressed consistently in tumour cells of both familial and sporadic basal cell carcinoma. Cancer Res 57:2336-2340

38. Xie J, Murone M, Luoh SM et al (1998) Activating Smoothened mutations in sporadic basal-cell carcinoma. Nature 391:90-92

39. Taylor MD, Liu L, Raffel C et al (2002) Mutations in SUFU predispose to medulloblastoma. Nat Genet 31:306-310

40. Grachtchouk M, Mo R, Yu S et al (2000) Basal cell carcinomas in mice overexpressing Gli2 in skin. Nat Genet 24:216-217

41. Nilsson M, Unden AB, Krause D et al (2000) Induction of basal cell carcinomas and trichoepitheliomas in mice overexpressing GLI-1. Proc Natl Acad Sci U S A 97:3438-3443

42. Oro AE, Higgins KM, Hu Z et al (1997) Basal cell carcinomas in mice overexpressing sonic hedgehog. Science 276:817-821

43. Aszterbaum M, Epstein J, Oro A et al (1999) Ultraviolet and ionizing radiation enhance the growth of BCCs and trichoblastomas in patched heterozygous knockout mice. Nat Med 5:1285-1291

44. Rubin LL, de Sauvage FJ (2006) Targeting the Hedgehog pathway in cancer. Nat Rev Drug Discov 5:1026-1033 